\title{
ARTIGOS
}

\section{SENSIBILIDADE E ESPECIFICIDADE DO DIAGNÓSTICO CLÍNICO, SOROLÓGICO E TOMOGRÁFICO DA ENCEFALITE POR TOXOPLASMA GONDII NA SÍNDROME DA IMUNODEFICIÊNCIA ADQUIRIDA (SIDA)}

\author{
Marco V. Wainstein, Luciano Wolffenbuttel, Demetrius K. Lopes, Helena E. \\ González, Liane Golbspan, Lisiane Ferreira, Eduardo Sprinz, Matias \\ Kronfeld e Maria I. Edelweiss
}

\begin{abstract}
A encefalite por Toxoplasma gondii (ET) é a principal causa de massa no sistema nervoso central (SNC) em pacientes com a sindrome da imunodeficiência adquirida (SIDA). Com o objetivo de determinar a prevalência dessa afecção e da presença de anticorpos específicos no soro e no liquor, bem como a sensibilidade (S) e a especificidade (E) da tomografia computadorizada (TC), dos achados clínicos e dos testes especificos foram revisados todos os prontuários de 516 pacientes com SIDA, internados no HCPA entre maio/85 e dezembro/91. A prevalência através de TC foi de $13 \%$ (diagnóstico presuntivo). A pesquisa de anticorpos especificos para toxoplasmose por imunofluorescência indireta no sangue (SS) foi positiva em $65 \%$ e no liquor (SL) em $49 \%$ dos pacientes em que foi realizada. Necrópsias de 125 pacientes foram revisadas encontrando-se uma prevalência de ET em 27 (22\%) casos, em que o diagnóstico foi considerado definitivo. A sensibilidade da TC foi de $65 \%$ e a especificidade de $82 \%$. A SS apresentou $S$ de $95 \%$ e E de 30\%, enquanto a SL apresentou uma $S$ de $77 \%$ e $E$ de $56 \%$. Os seguintes achados clínicos foram pesquisados: febre $(S=92 \% ; E=56 \%)$; sinais neurológicos focais $(S=59 \% ; E=82 \%$ ) e cefaléia $(S=41 \% ; E=69 \%)$. Conclutmos que é alta a prevalência da ET na SIDA e que a TC e a pesquisa de anticorpos especificos no soro e no liquor, devido da alta especificidade da primeira e a alta sensibilidade da segunda, constituemse ein métodos adequados para o diagnóstico da ET, sendo discutivel a necessidade de realizar biopsia cerebral nesses casos.
\end{abstract}

Palavras-chaves: SIDA. Encefalite por Toxoplasma gondii. Prevalência.

A encefalite por Toxoplasma gondii (ET) é uma das mais freqüentes afecções do sistema nervoso central (SNC) e a principal causa de lesão expansiva em pacientes com SIDA 17811151720 , apresentando prevalência de 3 a $40 \%$ nesses pacientes ${ }^{711} 12$. Em nossos dois estudos anteriores, demonstramos através de achados de necrópsias que a ET é a principal causa de lesão no SNC na SIDA, em nosso meio ${ }^{16}$; entretanto, a prevalência da doença por achados tomográficos era de apenas $10 \%^{19}$.

\footnotetext{
Trabalho realizado na Unidade de Doenças Infecciosas e Serviço de Anatomia Patológica do Hospital de Clínicas de Porto Alegre (HCPA), Porto Alegre, RS.

Auxílio: $\mathrm{CNPq}$

Endereço para correspondência: Dra. Maria I. Edelweiss. Serviço de Anatomia Patológica/HCPA. R. Ramiro Barcelos 2350 subsolo, 93054-490 Porto Alegre, RS, Brasil.

Recebido para publicação em $30 / 12 / 92$.
}

Existe controvérsia a respeito da importância da sorologia antitoxoplásmica para realização do diagnóstico de ET em pacientes com SIDA, uma vez que a doença resulta da reativação de foco de infecção latente, não constituindo manifestação de doença aguda $^{9}$. No entanto, a ausência de anticorpos antiToxoplasma gondii da classe IgG em pacientes com suspeita de ET, depõe fortemente contra esse diagnóstico ${ }^{6}$.

A tomografia computadorizada (TC) contrastada tem demonstrado ser bastante útil para o diagnóstico de ET, revelando tanto lesões anulares quanto nodulares em $90 \%$ dos pacientes ${ }^{14}$. Através de análise bayesiana demonstrou-se que pacientes, que apresentam lesões compatíveis com o diagnóstico de toxoplasmose na TC, associados com a presença de títulos sorológicos de imunoglobulinas específicas da classe IgG no sangue acima de 1:64, possuem $81 \%$ de probabilidade de desenvolverem $\mathrm{ET}^{3}$. Dessa 
Wainstein MV, WolffenbuttelL, Lopes DK, González HE, Golbspan L, Ferreira L, Sprinz E, Kronfeld M, Edelweiss MI. Sensibilidade e especificidade do diagnóstico clínico, sorológico e tomográfico da encefalite por Toxoplasma gondii na síndrome da imunodeficiência adquirida (SIDA). Revista da Sociedade Brasileira de Medicina Tropical 26:71-75, abr-jun, 1993.

forma, tem-se admitido que odiagnóstico presuntivo de ET pode ser realizado em pacientes que apresentam lesões expansivas na TC e anticorpos específicos no soro, mesmo na ausência de sinais ou sintomas e alterações tomográficas e sorológicas patognomônicas de ET. O objetivo deste estudo é determinar a prevalência da ET em nosso meio, bem como avaliar a sensibilidade e a especificidade da tomografia e da pesquisa de anticorpos específicos para Toxoplasma gondii, tendo em vista a realização desse diagnóstico.

\section{MATERIAL E MÉTODOS}

Foram revisados os prontuários de 516 pacientes com SIDA (de acordo com critérios do CDC, 1987), internados no Serviço de Doenças Infecciosas do Hospital de Clínicas de Porto Alegre (HCPA), entre maio/85 e dezembro/91. Em 125 casos submetidos à necrópsia, os cérebros foram submetidos a exame anatomopatológico.

Os prontuários foram revisados em relação a achados clínicos, tomográficos e pesquisa de anticorpos especificos coptra Toxoplasma gondii, por imunofluorescência indireta, no sangue e no líquor. Se o paciente apresentasse vários diferentes títulos desses anticorpos, em vários exames, o título do teste realizado em data mais próxima da TC foi levado em consideração; em caso de não haver sido realizada TC, o maior título foi levado em conta no estudo. Considerou-se como diagnóstico presuntivo de ET, neste trabalho, a presença de uma TC revelando lesões contrastadas anulares e/ou nodulares hipodensas ou hiperdensas com edema e efeito de massa.

O diagnóstico definitivo de ET foi realizado pela presença de cistos ou trofozoítos livres de Toxoplasma gondii em cortes histológicos cerebrais ou através de achados de necrópsia compativeis com toxoplasmose cerebral. Foram estabelecidos três padrões morfológicos diferentes de envolvimento cerebral: abscesso necrotizante com escassa resposta inflamatória e numerosos taquizoítos; abscesso limitado circundado por macrófagos e linfócitos com número variável de taquizoítos; e abscesso crônico, com nódulos e raros cistos e taquizoítos. Todos os cortes cerebrais foram examinados macro e microscopicamente, rotineiramente, através de hematoxilina-eosina e, quando necessário, empregando-se coloraçōes especiais como PAS, Grocott, Giemsa e ZiehlNielsen.

\section{RESULTADOS}

Durante o período de maio/85 e dezembro/91, 516 pacientes foram incluídos no estudo, dos quais apenas 44 eram do sexo feminino. A prevalência de ET estabelecida por TC (diagnóstico presuntivo), o diagnóstico definitivo realizado por necrópsia e os resultados do teste de imunofluorescência indireta para o diagnóstico de toxoplasmose, no sangue e no líquor, estão resumidos na Tabela 1 . O diagnóstico definitivo de ET foi firmado em 27 (22\%) dos 125 pacientes submetidos a necrópsia.

A TC cerebral foi realizada em 209 pacientes, sendo compatível com ET em 66 (31\%); considerando-se todos os 516 pacientes, incluídos

Tabela 1 - Prevalência de alterações encontradas na necrópsia, na tomografia computadorizada (TC), e no teste de imunofluorescência indireta (IF) para toxoplasmose em pacientes com SID.1.

\begin{tabular}{lcccc}
\hline Exame & $\begin{array}{c}\text { Total } \\
\text { de } \\
\text { casos }\end{array}$ & $\begin{array}{c}\text { Número de pacientes } \\
\text { estudados }\end{array}$ & $\begin{array}{c}\text { Número de casos } \\
\text { com alterações } \\
\text { sugestivas de } \\
\text { toxoplasmose }\end{array}$ & $(\%)$ \\
\hline $\begin{array}{l}\text { Necrópsia } \\
\text { TC* }\end{array}$ & 516 & 125 & 27 & 22 \\
$\begin{array}{l}\text { IF toxoplasmose } \\
\text { no sangue }\end{array}$ & 516 & 209 & 66 & 13 \\
$\begin{array}{l}\text { IF toxoplamose } \\
\text { no líquor }\end{array}$ & 516 & 343 & 225 & 65 \\
\hline
\end{tabular}

* A prevalência na TC é baseada no número total de casos (516). 
Wainstein MV, WolffenbuttelL, Lopes DK, González HE, Golbspan L, Ferreira L, Sprinz E, Kronfeld M, Edelweiss MI. Sensibilidade e especificidade do diagnóstico clínico, sorológico e tomográfico da encefalite por Toxoplasma gondii na sindrome da imunodeficiência adquirida (SIDA). Revista da Sociedade Brasileira de Medicina Tropical 26:71-75, abr-jun, 1993.

no estudo, o diagnóstico presuntivo de ET alcançou uma prevalência de $13 \%$. A sensibilidade da TC atingiu $65 \%$, enquanto a especificidade foi de $82 \%$ e os valores preditivos positivo e negativo foram, respectivamente, $68 \%$ e $80 \%$ (Tabela 2 ).

$O$ teste de imunofluorescência (IF) para toxoplasmose foi positivo no soro de $225(65 \%)$ dos 343 pacientes em que foi realizado; todos os 225 tinham anticorpos específicos da classe IgG, mas em apenas três estavam presentes anticorpos da classe IgM. A sensibilidade e a especificidade da IF no soro foram, respectivamente, de $95 \%$ e $30 \%$. Quando considerados apenas os títulos de anticorpos maiores que 1:1000, a sensibilidade e a especificidade corresponderam, respectivamente, a $62 \%$ e $77 \%$ (Tabela 2). A IF no líquor foi positiva em 83 (49\%) dos 169 pacientes em que foi realizada; em nenhum desses casos foram detectados anticorpos da classe IgM. Quando comparados os resultados da IF no soro e no líquor, observou-se uma sensibilidade menor especificidade maior no líquor, correspondentes, respectivamente, a $77 \%$ e $56 \%$.

Três dos achados clínicos que se associaram com maior freqüência com a ET (febre - temperatura axilar acima de $37,5^{\circ} \mathrm{C}$, sinais neurológicos focais e cefaléia) foram por nós avaliados. A febre apresentou a maior sensibilidade e a menor especificidade, correspondendo, respectivamente, a $92 \%$ e $7 \%$. Os sinais neurológicos focais apresentaram $59 \%$ de sensibilidade e $82 \%$ de

\section{Tabela 2 - Sensibilidade e especificidade da tomografia computadorizada (TC), do teste de imunofluorescência indireta (IF) para toxoplasmose no soro e no líquor e dos achados clínicos para o diagnóstico de encefalite por Toxoplasma gondii (ET).}

\begin{tabular}{lcc}
\hline Exame/dados clínicos & $\begin{array}{c}\text { Sensibilidade } \\
(\%)\end{array}$ & $\begin{array}{c}\text { Especifi- } \\
\text { cidade (\%) }\end{array}$ \\
\hline TC & 65 & 82 \\
IF no soro & 95 & 30 \\
Títulos > 1000 & 62 & 77 \\
IF no líquor & 77 & 56 \\
TC + títulos > 1000 da IF* & 83 & 59 \\
Febre & 92 & 07 \\
Sinais neurológicos & 59 & 82 \\
Cefaléia & 41 & 69 \\
\hline
\end{tabular}

* Títulos $>1000$ na sorologia sanguínea. especificidade, enquanto a cefaléia demonstrou sensibilidade de $41 \%$ e especificidade de $69 \%$ (Tabela 2).

\section{DISCUSSÃO}

Os resultados encontrados não deixam dúvidas quanto ao fato de que a encefalite por Toxoplasma gondii na SIDA é uma doença de grande importância e alta prevalência em nosso meio. A prevalência do diagnóstico definitivo estabelecida através de necrópsia (22\%) é compatível com os registros da literatura 561213 . É, no entanto, provável que essa prevalência seria ainda maior se técnicas de imunohistoquímica tivessem sido empregadas nas necrópsias ou se tivessem sido considerados os casos de sucesso terapêutico para fins diagnósticos.

$\mathrm{Na}$ maioria dos estudos, evidencia-se alta prevalência da ET na SIDA, com base em achados tomográficos, embora esse critério permita apenas diagnóstico presuntivo. A prevalência da ET através de TC, em nosso estudo, foi de somente $13 \%$. Em estudo retrospectivo de TC cerebrais em pacientes com SIDA, demonstrou-se que a TC é bastante sensível para detecção de lesões cerebrais causadas por Toxoplasma gondii, já que todas as TC foram anormais ${ }^{14}$. Em nosso trabalho, a TC apresentou sensibilidade razoável $(65 \%)$, embora sua especificidade tenha sido muito elevada (82\%). Esse achado pode estar relacionado com o padrão diagnóstico bastante restrito considerado na TC neste estudo.

A maior parte dos pacientes com ET apresentam, cefaléia, febre, hemiparesia leve, confusão mental ou convulsões focais, e menos freqüentemente, alterações de motricidade ${ }^{101318}$. O mais específico dos achados clínicos em nosso estudo foram os sinais neurológicos focais (82\%). A febre demonstrou ser indice de alta sensibilidade (92\%) e a cefaléia revelou sensibilidade e especificidade intermediárias, correspondentes, respectivamente, a $41 \%$ e $69 \%$.

O significado da IF para toxoplasmose realizada no soro, para diagnóstico de ET, permanece alvo de controvérsia. $O$ fato de somente três dos pacientes com sorologia sanguínea positiva apresentarem anticorpos específicos da classe IgM está de acordo com a literatura ${ }^{1018}$, confirmando a idéia de que a ET representa reativação de infecção latente. 
Wainstein MV, WolffenbuttelL, Lopes DK, González HE, Go.bspan L, Ferreira L, Sprinz E, Kronfeld M, Edelweiss MI. Sensibilidade e especificidade do diagnóstico clínico, sorológico e tomográfico da encefalite por Toxoplasma gondii na sindrome da imunodeficiência adquirida (SIDA). Revista da Sociedade Brasileira de Medicina Tropical 26:71-75, abr-jun, 1993.

Segundo alguns autores ${ }^{20}$, seria difícil estabelecerse o diagnóstico de ET com base em achados sorológicos. Os mesmos autores admitem que a ausência de títulos de anticorpos IgG elevados não afasta a possibilidade de ET em pacientes com SIDA. Nossos achados sorológicos estão de acordo com a idéia de que os títulos encontrados não são diagnósticos de ET mas, em virtude de sua alta sensibilidade ( $95 \%$ ), consideramos que a ausência de anticorpos especificos contra o Toxoplasma gondii constitui informação importante para excluir a possibilidade de ET. Na tentativa de aumentar a acurácia diagnóstica, através de testes seriados, selecionamos os pacientes que apresentavam TC compatível com o diagnóstico de ET e títulos de anticorpos no soro acima de 1:1000, encontrandose sensibilidade de $83 \%$ e especificidade de $59 \%$, o que ressalta a importância de associar esses dois métodos diagnósticos.

Em relação ao teste de IF para toxoplasmose realizado no líquor, sabe-se que número significativo de pacientes é capaz de apresentar anticorpos nesse líquido orgânico ${ }^{11}$. A produção local de anticorpos pode constituir indicador específico de ET, mas aproximadamente $50 \%$ desses pacientes não irão apresentar anticorpos detectáveis no líquor. Isto foi confirmado em nosso estudo, no qual os resultados no líquor tiveram maior especificidade $(56 \%)$ do que no soro $(30 \%)$. O motivo pelo qual alguns pacientes com ET não produzem anticorpos antitoxoplasma, localmente, permanece obscuro.

Existem dúvidas a respeito da necessidade e da utilidade da biópsia cerebral para o diagnóstico de $\mathrm{ET}^{11}$. A biópsia deve ser realizada em pacientes com SIDA nos quais outras causas de encefalite possam ser freqüentes. Segundo Grant e $\operatorname{cols}^{5}$, a realização de biópsia deve ser considerada em pacientes que não responderam à terapia específica no fim de uma a duas semanas, ou mesmo antes, se o paciente se encontra sob alto risco de desenvolver abscesso bacteriano. A possibilidade de outros agentes infecciosos e neoplasias mimetizarem perfeitamente a apresentação clínica de ET tem induzido alguns autores a recomendar a realização de biópsia em todas as lesões expansivas do $\mathrm{SNC}$ em pacientes com SIDA ${ }^{2814}$. A imediata resposta ao tratamento clínico adequado tem, entretanto, levado alguns autores ${ }^{4}$ a sugerir a introdução de tratamento empírico em pacientes com SIDA que apresentam lesões compatíveis com toxoplasmose cerebral na $\mathrm{TC}$, nos quais não existe evidência de outra etiologia. Esse achado foi confirmado em estudo onde os pacientes com lesões contrastadas na $\mathrm{TCe}$ sorologia antitoxoplásmica positiva apresentaram um valor preditivo positivo de $81 \%$ para o diagnóstico de ET. Esse subgrupo de pacientes foi considerado como excelente candidato para terapia empírica, em função da alta probabilidade de apresentarem a doença ${ }^{3}$.

Com base na alta sensibilidade da sorologia sanguínea (95\%) e na alta especificidade da TC $(82 \%)$, demonstradas neste estudo, podemos estabelecer o diagnóstico de ET quando a TC apresentar alterações compatíveis com toxoplasmose cerebral. Por outro lado, teste sorológico para toxoplasmose com resultado negativo (ausência de anticorpos) torna improvável o diagnóstico de ET. Portanto, de acordo com nossos resultados, podemos sugerir o tratamento empírico para ET em presença de TC com alterações compatíveis, associada com positividade do teste sorológico para toxoplasmose.

\section{SUMMARY}

Toxoplasmic encephalitis (TE) is among the most common neurologic affections and it is the most prevalent cause of intracerebral mass lesions in AIDS patients. All patients with AIDShospitalized at the Hospitalde Clinicas de Porto Alegre between May/85 and December/91 (516 cases) had theirfiles revised to determinate TE prevalence, serology, sensivity and specificity of the computed tomography (CT) brain scan, clinical findings and serology to make its diagnosis. The prevalence on CT was $13 \%$ (presumptive diagnosis). Blood serology and cerebrospinal (CSF) serology to toxoplasma were positive respectively in $65 \%$ and $49 \%$. Autopsies of 125 patients were also revised with a prevalence of $22 \%$ (definite diagnosis). CT scan had $65 \%$ of sensivity and $82 \%$ of specificity. Sensivity and specificity of serology on blood was respectively $95 \%$ and $30 \%$, while the cerebrospinal fluid (CSF) serology had 77\% of sensivity and $56 \%$ of specificity. The following clinical findings were considered: fever (sensivity $=92 \%$; specificity $=56 \%$ ), neurological focal signs (sensivity $=59 \%$; specificity $=82 \%$ ) and headache (sensivity $=41 \%$; specificity $=69 \%$ ). We conclude that, based on the high serology sensivity and high CT scan specificity, they constitute an useful approach to make TE diagnosis.

Key-words: Toxoplasmic encephalitis. AIDS. Prevalence. 
Wainstein MV, Wolffenbuttel L, Lopes DK, González HE, Golbspan L, Ferreira L, Sprinz E, Kronfeld M, Edelweiss MI. Sensibilidade e especificidade do diagnóstico clínico, sorológico e tomográfico da encefalite por Toxoplasma gondii na sindrome da imunodeficiência adquirida (SIDA). Revista da Sociedade Brasileira de Medicina Tropical 26.71-75, abr-jun, 1993.

\section{REFERÊNCIAS BIBLIOGRÁFICAS}

1. Anders KH, Guerra WF, Tomiyasu U, Verity MA, Vinters HV. The neuropathology of AIDS: UCLA experience and review. American Journal of Pathology 124:537-558, 1986.

2. Bishburg E, Slim J, Johnson ES, Kapita R, Eng RHK. The need for tissue diagnosis of central nervous system lesions with the acquired immunodeficiency syndrome. In: Abstract of Proceedings of the Third International Conference on AIDS, Washington, DC p.36, 1987.

3. Cimino C, Lipton RB, Williams A, Feraru E, Harris C, Hirschfeld A. The evaluation of patients with human immuno-deficiency virus-related disorders and brain mass lesions. Archives of Internal Medicine 151:1381-1384, 1991.

4. Cohn JA, McMeeking A, Cohen W, Jacobs J, Holzman RS. Evaluation of the policy of empiric treatment of suspected Toxoplasma encephalitis in patients with the acquired immunodeficiency syndrome. American Journal of Medicine 86:521527, 1989.

5. Grant IH, Gold JW, Rosenblum M, Niedzwiecki D, Armstrong D. Toxoplasma gondii serology in HIVinfected patients: the development of central nervous system toxoplasmosis in AIDS. AIDS 4:519-521, 1990.

6. Israelski DM, Dannemann BR, Remington JS. Toxoplasmic encephalitis in patients with AIDS. In: Sande MA, Volverding PA (eds) The medical management of AIDS, WB Saunders, Philadelphia p.241-266, 1990.

7. Levy RM, Bredsen DE, Rosenblum ML. Neurological manifestations of the acquired immunodeficiency syndrome (AIDS): experience at UCSF and review of the literature. Journal of Neurosurgery 621:475-495, 1985.

8. Levy RM, Pons VG, Rosenblum ML. Central nervous system mass lesions in acquired immunodeficiency syndrome (AIDS). Journal of Neurosurgery 61:9-16, 1984.

9. Luft BJ, Brooks RG, Conley FK, McCabe RE, Remington JS. Toxoplasmic encephalitis in patients with acquired immunodeficiency syndrome. Journal of the American Medical Association 252:913-917, 1984.

10. Luft BJ, Conley F, Remington JS, Laverdiere $M$, Levine JF, Strandberg DA, Wagner KF, Craven PC, File TM, Rice N, Carpentier FM. Outbreak of central-nervous-system toxoplasmosis in western Europe and North America. New England Journal of Medicine 1:781-783, 1983

11. Luft BJ, Remington JS. AIDS commentary: toxoplasmic encephalitis. Journal of Infectious Diseases 157:1-6, 1988.

12. Luft BJ, Remington JS. Toxoplasmosis of the central nervous system. In: Remington JS, Swartz MN (eds) Current clinical topics in infectious diseases. McGraw-Hill, New York p.315-358, 1985.

13. Navia BA, Petito CK, Gold JW, Cho ES, Jordan $\mathrm{BD}$, Price RW. Cerebral toxoplasmosis complicating the acquired immunodeficiency syndrome: clinical and neurophatological findings in 27 patients. Annals of Neurology 19:224-238, 1986.

14. Post MJD, Kursunoglu SJ, Hensley GT, Chan JC, Moskowitz LB, Hoffman TA. Cranial CT in acquired immunodeficiency syndrome; spectrum of diseases and optimal contrast enhancement technique. American Journal of Radiology 145:929-940, 1985.

15. Snider WD, Simpson DM, Nielsen S, Gold JW, Metroka CE, Posner JB. Neurological complications of acquired immunodeficiency syndrome: analysis of 50 patients. Annals of Neurology 14:403-418, 1983.

16. Wainstein MV, Ferreira L, Wolffenbuttel L, Golbspan L, Sprinz E, Kronfeld M, Edelweiss MI. Achados neuropatológicos na síndrome da imunodeficiência adquirida (SIDA): revisão de 138 casos. Revista da Sociedade Brasileira de Medicina Tropical 25:95-99, 1992

17. Whelan MA, Kricheff II, Handler M. Acquired immunodeficiency deficiency syndrome: cerebral computed tomographic manifestations. Radiology 149:477-484, 1983.

18. Wijdicks EM, Borleffs JC, Hoepelman AM, Jansen GH. Fatal disseminated hemorrhagic toxoplasmic encephalitis as the initial manifestation of AIDS. Annals of Neurology 29:683-686, 1991.

19. Wolffenbuttel L, Wainstein MV, Ferreira L, Lubianca JF, Petrillo VF, Kronfeld M. Prevalência da encefalite por toxoplasma em pacientes com SIDA no Hospital de Clínicas de Porto Alegre. Revista da Associação Médica do Rio Grande do Sul 35:23-26, 1991.

20. Wong B, Gold JW, Brown AE, Lange M, Fried R, Grieco M, Mildvan D, Giron J, Tapper ML, Lerner CW, Armstrong D. Central-nervous-system toxoplasmosis in homosexual men and parenteral drug abusers. Annals of Internal Medicine 100:36$42,1984$. 